\section{Proton Beam Therapy}

\author{
Carol L. Armstrong
}

Child and Adolescent Psychiatry and Behavioral

Sciences, The Children's Hospital of

Philadelphia, Philadelphia, PA, USA

\section{Synonyms}

Proton radiation therapy; Proton therapy

\section{Definition}

Proton beam therapy is another form of ionizing radiation therapy used for mass cancer lesions. It was first proposed by Robert Wilson in 1946 and is increasingly available though much more expensive than photon radiotherapy. It uses subatomic particles instead of photons, and more energy is deposited in the Bragg Peak. Protons have the property of increasing the dose very gradually with increasing depth, and then rising to a peak at the end of its range (Bragg Peak), thus delivering less radiation in front of the tumor and no radiation behind the tumor. Studies have indicated it is at least as effective as photon radiotherapy and the toxic risks are no greater (Giantsoudi et al. 2016). No prospective results on cognitive functions are yet available. Early findings indicate no difference in the slope of change in IQ over time in children (Kahalley et al. 2016).
Proton beam therapy is most often used in treatment of tumors of the brain, head, neck, lungs, and prostate. In conventional radiotherapy, photons or electrons are used, though the biological effects of protons are similar (Glimelius and Montelius 2007). It is more precise than other forms of radiotherapy because radiation targeted at the tumor cavity does not go below or beyond the tumor, thus reducing the amount of radiation that is absorbed by healthy tissue, and allowing a higher dose to the tumor. It can be used in multiple fractions over several weeks, or as radiosurgery.

\section{References and Readings}

Armstrong, C. L., Schmus, C. J., \& Belasco, J. B. (2011). Neuropsychological problems in neuro-oncology. In C. L. Armstrong \& L. Morrow (Eds.), Handbook of medical neuropsychology: Applications of cognitive neuroscience. New York: Springer.

Giantsoudi, D., Sethi, R. V., Yeap, B. Y., Eaton, B. R., Ebb, D. H., Caruso, P. A., et al. (2016). Incidence of CNS injury for a cohort of 111 patients treated with proton therapy for medulloblastoma: LET and RBE associations for areas of injury. International Journal of Radiation Oncology, Biology, and Physics, 95(1), 287-296.

Glimelius, B., \& Montelius, A. (2007). Proton beam therapy - Do we need the randomised trials and can we do them? Radiotherapy and Oncology, 83, 105-109.

Kahalley, L. S., Ris, M. D., Grosshans, D. R., Okcu, M. F., Paulino, A. C., Chintagumpala, M., et al. (2016). Comparing intelligence quotient change after treatment with proton versus photon radiation therapy for pediatric brain tumors. Journal of Clinical Oncology, 34(10), 1043-1049. 